\title{
Clustering Opportunistic Ant-based Routing Protocol for Wireless Sensor Networks
}

\author{
Xinlu Li ${ }^{1}$ \\ Department of Computer Science, Hefei University \\ Hefei, China \\ School of Computing, Dublin Institute of Technology \\ Dublin, Ireland \\ E-mail: xinlu.li@mydit.ie

\section{Brian Keegan} \\ School of Computing, Dublin Institute of Technology \\ Dublin, Ireland \\ E-mail: brian.x.keegan@dit.ie

\section{Fredrick Mtenzi} \\ School of Computing, Dublin Institute of Technology \\ Dublin, Ireland \\ E-mail: Fredrick.Mtenzi@dit.ie
}

The wireless Sensor Networks (WSNs) have a wide range of applications in many ereas, including many kinds of uses such as environmental monitoring and chemical detection. Due to the restriction of energy supply, the improvement of routing performance is the major motivation in WSNs. We present a Clustering Opportunistic Ant-based Routing protocol (COAR), which comprises the following main contributions to achieve high energy efficient and well load-balance: (i) in the clustering algorithm, we caculate the theoretical value of energy dissipation, which will make the number of clusters fluctuate around the expected value, (ii) define novel heuristic function and pheromone update manner, develop an improved ant-based routing algorithm, in this way, the optimal path with lower energy level and shorter link length is established, and (iii) propose the energy-based opportunistic broadcasting mechanism to reduce the routing control overhead. We implement COAR protocol in NS2 simulator and our extensive evaluation shows that COAR is superior to some seminal routing algorithms under a wide range of scenarios.

CENet 2017

22-23 July, 2017

Shanghai, China

\footnotetext{
${ }^{1}$ This research is supported by the Science Foundation for Youths of Hefei University (Grant No. 16YQ08RC) 


\section{Introduction}

Internet of Things (IoT) has become a rapid growth industry, wireless sensor networks(WSNs), as the basis of IoT, has become a hot spot for application and research as the same time. WSNs consist of a large number of distributed sensor nodes[1]. These sensor nodes usually have many limitations in terms of energy supply, computing and communication abilities, hence the core feature of the wireless sensor routing protocol is the energy efficency; in other words, the protocol can extend the network lifetime and improve the quality of network communications. There are many current works, and efforts that are ongoing for the development of the energy-efficient routing protocols in WSNs.

As the classical protocols, the hierarchical routing algorithm is very promising and thus has received a lot of attentions of the researchers, who utilized several schemes to select node clusters and fuse the sensor data in each cluster and then relay the data towards the base station [2-5]. These routing protocols tried to achieve better network lifetime by using cluster-based routing algorithm and specific data fusion procedure; however, most of these cluster-based routing protocols $[3,5-7]$ randomly select the Cluster Head nodes (CHs). These stochastically selected cluster heads method will result in the division of heterogeneous and unblanced clusters in the network. Besides, the $\mathrm{CHs}$ are responsible for data collection and fusion in its cluster. When the size of the cluster is greater, the $\mathrm{CHs}$ would deplete more power and run out of energy quickly, finnally result in shorter lifetime or network hotspot [8-10].

Researchers have proposed a number of routing protocols by using Ant Colony Optimization (ACO) [11], and these protocols imitate the ant colonies foraging behavior to define a nature inspired metaheuristic to discover the route to the destination. The first ACO based routing protocol is presented for wired network [12], then for Ad Hoc networks (MANETs) [13]. Zhang et al. [14] found these protocols [12] and [13] were not suitable for sensor networks and then presented a foundation framework for ant based WSNs routing protocol. An Energy-Efficient Ant Base Routing Algorithm (EEABR) [15] considered the energy factor of wireless sensor node on the basis of ACO mechanism to extend network lifetime. Chen et al. presented the E-D ANTS [16] protocol which intended to find a path with minimum energy consumption to maximize the network lifetime. R. Khoshkangini et al. [17] combined ACO with breadth first search to discover the shortest path in order to improve data transmission with the least amount of energy consumption. Sun, Y et al. [18] improved heuristic function and considered the node communication transmission distance and residual energy to find the optimal path of data transmission. Our previous conference proposed an hierarchical ant colony based Routing (ACCR) [19] protocol for large scale wireless sensor networks. In ACCR, we present a clustering algorithm based on estimating average energy and an improved ant colony optimization based routing protocol. The preliminary simulation results show that COAR exhibits good performance in terms of energy efficiency and network lifetime.

We make several significant improvements on the basis of our previous work andthe main contributions include: (1) the improvement of the pheromone update strategy; (2) the development of an opportunistic broadcast scheme, which adopts normalized energy as link quality. Secondly, we simulate COAR in a large range application scenarios to evaluate the performance in terms of energy efficiency and characteristics of clusters, compare with seminal routing protocols. 


\section{Related Works}

In hierarchical routing model, Cluster Heads play an important role in hierarchical algorithm [20]. Some CHs selection algorithms [2, 3, 5-7] didn't take the residual energy of node into account, in that case, the node with lower energy may be selected to be $\mathrm{CH}$. Some other algorithms introduced the residual energy into selection threshold, but these clustering algorithms $[3,8,9,22]$ required too frequent message exchange related to energy, and then increased sharply the control overhead of the network, which would cause frequent network collision and additional energy consumption.

Plenty of ACO based routing algorithms were proposed to satisfied the shortest path [14] or the minimum energy consumption [15-17], and didn't consider other energy measures (e.g. average energy, maximum energy) [18], which would lead to some nodes with lower energy in optimal path exhaust energy prematurely, and the energy consumption of the entire network would run out of balance [11]. Furthermore, most of ACO based routing protocols feature flat network structure and fail to consider data correlation, hence, these protocols are very simply structured and fastly executable, and they are relatively effective for small-scale networks. However, they suffer from a large amount of control packet overhead and lack of scalability, therefore, It is difficult for them to adapt to large-scale network deployment[23].

In WSNs, duo to the mobility of nodes, the change of network topology and the nature of ACO algorithm, the neighboring nodes need to exchange the pheromone or residual energy frequently. Almost all the routing algorithms adopt the broadcast mechanism which is a fast and simple method to deliver the control packets (i.e. ant agent, hello message) [1], [11] by flooding the network. However, the routing control overhead and network congestion will increase correspondingly and then cause extra energy dissipation due to the flooding process.

\section{Algorithm Improvement}

Assumed N sensor nodes are stochastically deployed in a rectangle area. The Base Station (BS) locate in the corner of the rectangle. To reduce and balance the network energy consumption, prolong the network lifetime, we present several improvements, including energy estimation based clustering algorithm and an improved ACO based routing algorithm. Besides, in order to avoid network flooding, we propose a simple broadcast mechanism based on opportunistic selection.

\subsection{Clustering Algorithm based on Estimating Energy}

In hierarchical routing algorithm, the Cluster Heads are selected in each round according to a specified probability $p_{\text {opt }}$. Each node independently creates a random number in $[0,1]$. If the random number is less than a threshold $T(s)$, then the node becomes a $\mathrm{CH}$ in the current round.

$$
T(s)=\left\{\begin{array}{cc}
\frac{p_{o p t}}{1-p_{o p t}\left(r / \bmod \overline{p_{\text {opt }}}\right)} & , s \in G \\
0 & , \text { otherwise }
\end{array}\right.
$$

Where $r$ is the current round, $p_{\text {opt }}$ is an optimal Chs selection probability depending on the number of nodes in WSNs [2], and $G$ is the set of nodes that haven't been CHs in the last $1 / p_{o p t}$ rounds. 
We introduce an estimating average energy in round $r$,

$$
\bar{E}(r)=\frac{1}{N} E_{\text {total }}\left(1-\frac{r}{R}\right)
$$

Where $\mathrm{R}$ is the theoretical amount of round, $E_{\text {total }}$ denotes the total energy of network. $\bar{E}(r)$ denotes the estimating average energy. For more details, see our previous Paper [19]. Then we regard $\bar{E}(r)$ as the standard energy and define a desired probability for each node.

$$
p_{i}=p_{\text {opt }} \frac{E(i)}{\bar{E}(i)}
$$

Where $E_{i}$ is the residual energy, $\quad p_{\text {opt }}$ represents the optimal probability of becoming $\mathrm{CH}$ in each round, we use $p_{i}$ instead of $p_{\text {opt }}$ in threshold (3.1). By means of this improvement, the nodes with more residual energy will have more opportunities to become $\mathrm{CH}$, the energy consumption of the network will be evenly distributed correspondingly.

\subsection{Improved ACO based Routing Algorithm}

We develop an improved ant based routing algorithm using novel energy-related heuristic function and pheromone update manner. In COAR, we use this algorithm to deal with the communication between clusters. Each forward ant agent choice the next node based on a probabilistic function [19].

$$
p_{i j}(t)=\left\{\begin{array}{cc}
\frac{\tau_{i j}^{\alpha}(t) \times \eta_{i j}^{\beta}(t)}{\sum_{k} \tau_{i j}^{\alpha}(t) \times \eta_{i j}^{\beta}(t)} & , \forall j \in N_{j} \text { and } j \notin M^{k} \\
0 & , \text { otherwise }
\end{array}\right.
$$

$\tau_{i j}(t)$ and $\eta_{i j}(t)$ refer to the pheromone and heuristic information in link $(i, j)$ respectively, the coefficients $\alpha$ and $\beta$ represent their relative importance of $\tau_{i j}(t)$ and $\eta_{i j}(t)$. Here we redefine the $\eta_{i j}(t)$,

$$
\eta_{i j}(t)=\frac{E_{\text {init }}-\bar{E}(i)}{E_{\text {init }}-E(j)}
$$

Where $E_{\text {init }}-E(j)$ indicates the energy consumption of node $\mathrm{j}$, and $E_{\text {init }}-\bar{E}(j)$ represents the average energy consumption. By this way, the neighbor node with relatively less energy consumption features higher probability to forward the sensor data.

When the forward ant agent reaches the base station, the amount of pheromone increment $\Delta \tau(t)$ on the path is computed using such an improved manner:

$$
\Delta \tau(t)=\frac{E_{\min }}{\left(E_{\text {init }}-E_{\text {avg }}\right) F_{d}(t)}
$$

$E_{\text {min }}$ and $E_{\text {avg }}$ are the energy levels in the route towards base station. $F_{d}(t)$ implies the overall length of route. This manner ensures that the path with larger energy levels (i.e. minimum energy and average energy) and shorter distance will obtain a greater pheromone increment. Then the backward ant agent carries $\Delta \tau(t)$ updates the pheromone value along the reverse route according to following expression:

$$
\tau_{i j}(t)=(1-\rho) \tau_{i j}(t)+\Delta \tau_{i j}(t)
$$

Where $\rho \in(0,1)$ is the evaporation factor for each path, $\Delta \tau_{i j}(t)$ is calculated by,

$$
\Delta \tau_{i j}(t)=\frac{E(j)}{E_{\text {init }} B_{d}(t)} \Delta \tau(t)
$$


$B_{d}(t)$ indicates the route length from current node to base station. According to this method, the pheromone value depends on both the energy levels and the route length, therefore, the link with more residual energy will have more pheromone value, simultaneously the sensor nodes near the base station will have more pheromone, this positive mechanism will guide the sensor data through optimal path to the base staion and speed up the convergence rate.

\subsection{Opportunistic Broadcast}

As previously discussed in Section 2, the broadcast scheme is the easiest and most effective way to reach all the network nodes, which is widely used in ACO based routing protocols. We also use broadcast to transmit forward ant agent when it does not have available routing information. In broadcasting manner, the control message is duplicated in each node, then will worsen network performance, especially, when the network scale is large or frequent nodes mobility.

We improve the broadcast procedure using opportunistic routing concept. In this case, the procedure uses a opportunistic strategy to select a single neighbor node to re-broadcast the control message. Here we give a goodness function to denote the link quality $C(i, j)$ between the node $\mathrm{i}$ and its neighborhood $\mathrm{j}$.

$$
C(i, j)=\frac{E(i)-E_{\min }}{E_{\max }-E_{\min }}
$$

Where $E_{\min }$ and $E_{\max }$ are the minimum and maximum energy in the neighbors of node i. When receiving broadcast message, one of the neighborhoods will be picked up and rebroadcast the message by using a probabilistic approach.

$$
p_{i j}=\frac{C(i, j)}{\sum_{j \in M_{i}} C(i, j)}
$$

$M_{i}$ denotes the neighbors of node $\mathrm{i}$, and each node in neighborhood has probabilistic $p_{i j}$ to rebroadcast the control message. By this way, the opportunistic broadcast procedure will select different nodes at each time to re-broadcast the control message instead of flooding. Also, the node with more residual, i.e. with high link quality, have more chance to forward packet, which would reduce the overhead of the network and balance the energy consumption correspondingly.

\section{Simulation Results and Evaluation}

We compare the COAR protocol with the algorithms of LEACH [2], AntHocNet [13], EEABR [15] and SARA [24] in terms of characteristics of clusters, routing control overhead, energy efficiency, average energy consumption and energy standard deviation. We simulate these protocols under NS2, and in each case, 30 simulation runs are executed and $95 \%$ confidence intervals are achieved.

The number of clusters is the key characteristic in hierarchical model. There is an optimal clusters number $k_{\text {opt }}$, implying the clustering algorithm is stable [2]. Figure 1 indicates the clusters number $k$ of COAR and LEACH are fluctuating around the $k_{\text {opt }}$, but the volatility of COAR is fairly smooth, while the number of Chs in LEACH varies greatly due to desired probability strategy (3) for each node in COAR, which introduces the estimating average energy as standard value, however, LEACH usese the ordinary random manner. 


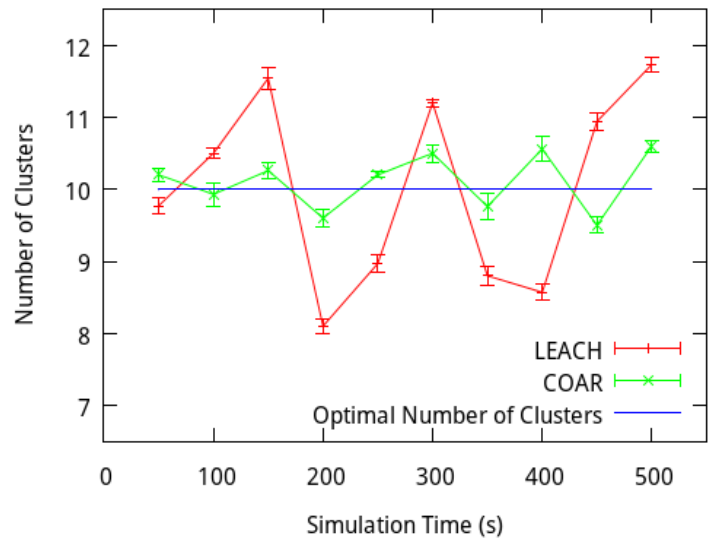

Figure 1:Characteristics of Clusters

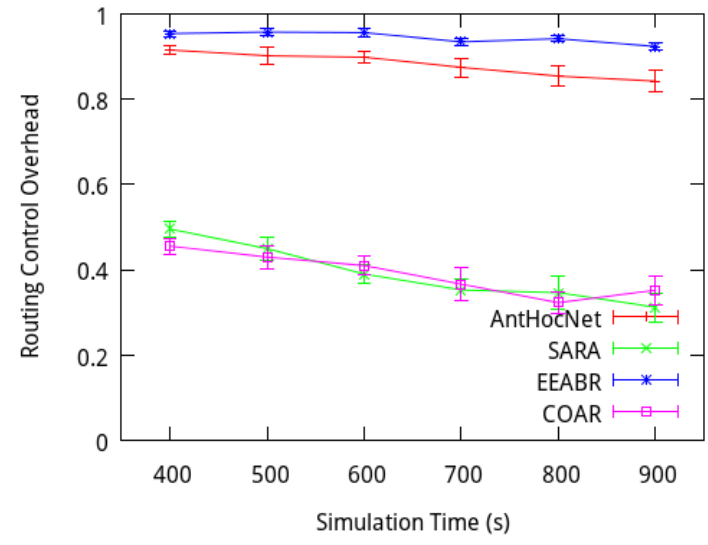

Figure 2: Routing Control Overhead

The routing control overhead measures the number of control message associated with the total amount of message that has been transmitted. High overhead would cause the network congestion and extra energy consumption. The comparison of routing control overhead is shown in Figure 2. Compared with the other protocols, COAR has the routing overhead ratio decreased by some extent and is similar to SARA, which is another protocol that uses the opportunistic broadcast algorithm. In COAR, each node rebroadcasts the control message with a given probability, which calculated according to the energy-related link quality. Consequently, the total amount of message forwarding is reduced significantly. In Figure 2, the overhead of COAR is smaller than that of AntHocNet and EEABR with \%50.

The average energy consumption is proportional with network lifetime. Figure 3 presents the results of different simulation times. As proved by the results, the COAR outperforms AntHocNet, EEABR and SARA protocols; and this improvement is mainly based on clustering routing algorithm and opportunistic broadcast scheme. In COAR, we get very close to the optimal number of clusters due to this clustering algorithm and data aggregation. The COAR achieves lower average energy consumption. Furthermore, COAR uses opportunistic broadcasting instead of flooding, which may reduce extra energy consumption for control message.

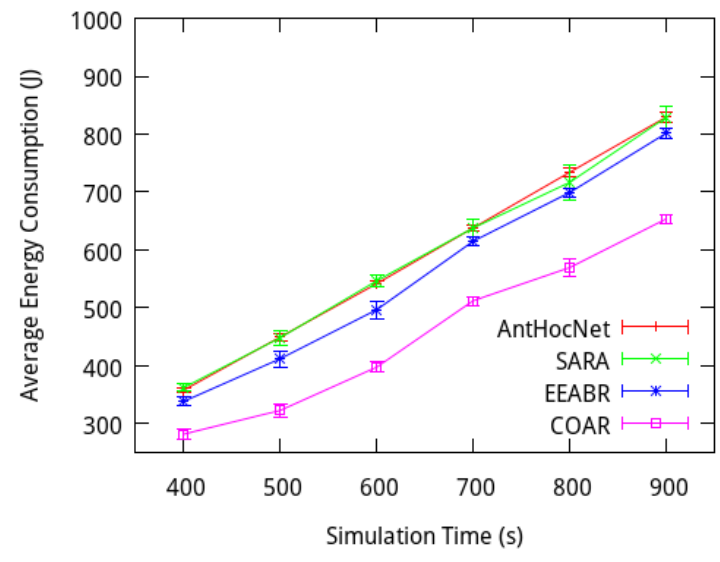

Figure 3: Average Energy Consumption

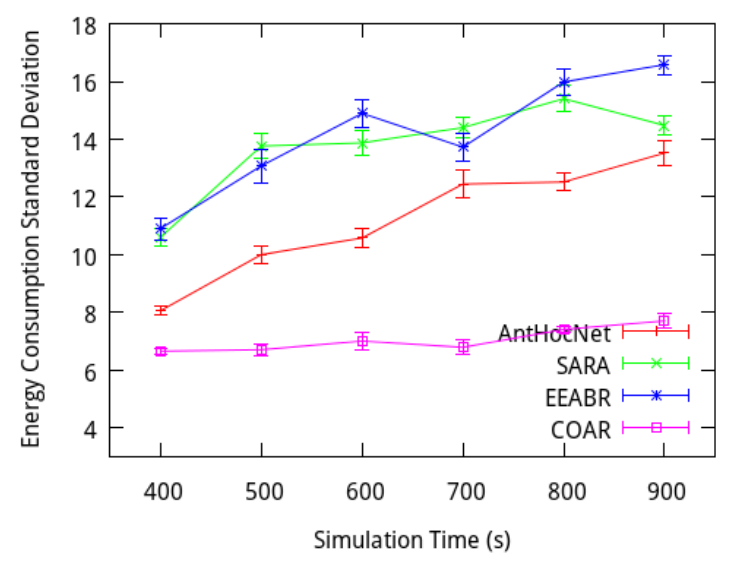

Figure 4:Energy Consumption Standard Deviation

The energy consumption standard deviation implies the average variance between energy consumed on all nodes. It describes the energy consumption distribution of the networks. A low standard deviation indicates that the data points tend to be close to the expected value of the set, 
while a high standard deviation indicates that the data points are spread out a wider range of values. Figure 4 illustrates that the standard deviation of COAR is always lower than that of AntHocNet, EEABR and SARA during the period of simulation, implying the energy consumption of COAR is distributed evenly due to the positive mechanism in ACO routing algorithm. COAR considers both energy levels and route length when updating the local heuristic and pheromone value. COAR finds the optimal route with higher energy and shorter length after several iterations, which results in well load-balance.

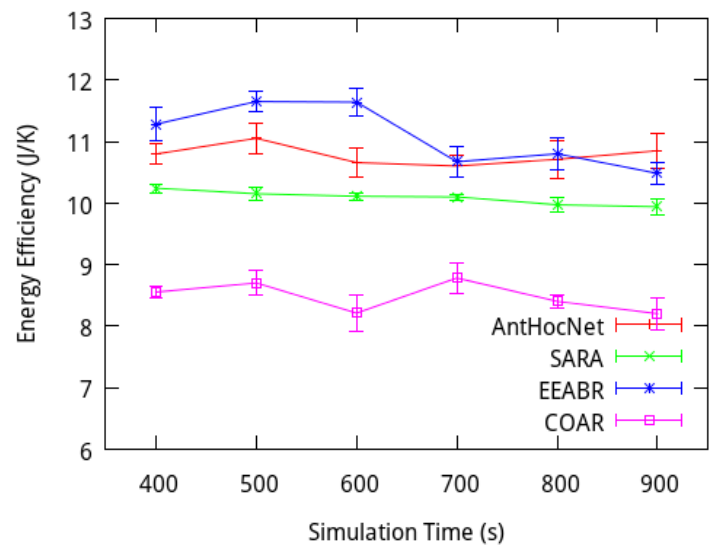

Figure 5: Energy Efficiency

The energy efficiency metric represents the energy required by the sensor node to transmit a sensor data packet to base staion. I Figure 5, COAR demonstrates great performance improvement over other protocols. Hence, the network could deliver more sensor data in case of limited power resources by using COAR protocol.

\section{Conclusion}

We propose an improved ACO clustering opportunistic routing protocol. COAR introduce a theoretical estimation energy dissipation as the standard energy in the route deterministic selection. The experiment results show that this hierarchical routing algorithm can reduce the average energy dissipation of the sensor network. Furthermore, we redefine the heuristic function and the manner of pheromone updating by considering both the energy levels and path length, which result in better load-balance and higher energy efficiency than other protocols, and then prolong the network lifetime. Besides, we introduce an energy-based opportunistic broadcast mechanism, which may significantly reduce the routing control overhead and correspondingly weaken the network congestion and extra energy dissipation.

\section{References}

[1] N. A. Pantazis, S. A. Nikolidakis, and D. D. Vergados, "Energyefficient routing protocols in wireless sensor networks: A survey," IEEE Communications surveys \& tutorials, vol. 15, no. 2, pp. 551-591, 2013.

[2] W. B. Heinzelman, A. P. Chandrakasan, and H. Balakrishnan, "An application-specific protocol architecture for wireless microsensor networks," IEEE Transactions on wireless communications, vol. 1, no. 4, pp. 660-670, 2002.

[3] A. Manjeshwar and D. P. Agrawal, "Teen: Arouting protocol for enhanced efficiency in wireless sensor networks.” in ipdps, vol. 1, 2001, p. 189. 
[4] Y. Liao, H. Qi, and W. Li, "Load-balanced clustering algorithm with distributed self-organization for wireless sensor networks," IEEE sensors journal, vol. 13, no. 5, pp. 1498-1506, 2013.

[5] G. Ahmed, J. Zou, M. M. S. Fareed, and M. Zeeshan, "Sleep-awake energy efficient distributed clustering algorithm for wireless sensor networks," Computers \& Electrical Engineering, 2015.

[6] P. A. Forero, A. Cano, and G. B. Giannakis, "Distributed clustering using wireless sensor networks," IEEE Journal of Selected Topics in Signal Processing, vol. 5, no. 4, pp. 707-724, 2011.

[7] D. Wei, Y. Jin, S. Vural, K. Moessner, and R. Tafazolli, “An energy-efficient clustering solution for wireless sensor networks," IEEE transactions on wireless communications, vol. 10, no. 11, pp. 3973-3983, 2011.

[8] A. Razaque, S. Mudigulam, K. Gavini, F. Amsaad, M. Abdulgader, and G. S. Krishna, "H-leach: Hybrid-low energy adaptive clustering hierarchy for wireless sensor networks," in Long Island Systems, Applications and Technology Conference (LISAT), 2016 IEEE., pp. 1-4.

[9] S. K. Gupta, P. Kuila, and P. K. Jana, "Energy efficient multipath routing for wireless sensor networks: A genetic algorithm approach," in Advances in Computing, Communications and Informatics (ICACCI), 2016 International Conference on. IEEE, 2016, pp. 1735-1740.

[10] M. Aslam, T. Shah, N. Javaid, A. Rahim, Z. Rahman, and Z. Khan, "Ceec: Centralized energy efficient clustering a new routing protocol for wsns," in Sensor, Mesh and Ad Hoc Communications and Networks (SECON), 2012 9th Annual IEEE Communications Society Conference on. IEEE, 2012, pp. 103-105.

[11] T. Gui, C. Ma, F. Wang, and D. E. Wilkins, "Survey on swarm intelligence based routing protocols for wireless sensor networks: An extensive study," in Industrial Technology (ICIT), 2016 IEEE International Conference on. IEEE, 2016, pp. 1944-1949.

[12] G. Di Caro and M. Dorigo, "Ant colony optimization and its application to adaptive routing in telecommunication networks," 2004.

[13] G. Di Caro, F. Ducatelle, and L. M. Gambardella, “Anthocnet: an ant-based hybrid routing algorithm for mobile ad hoc networks," in International Conference on Parallel Problem Solving from Nature. Springer, 2004, pp. 461-470.

[14] Y. Zhang, L. D. Kuhn, and M. P. Fromherz, "Improvements on ant routing for sensor networks," in International Workshop on Ant Colony Optimization and Swarm Intelligence. Springer, 2004, pp. 154-165.

[15] T. Camilo, C. Carreto, J. S. Silva, and F. Boavida, “An energyefficient ant-based routing algorithm for wireless sensor networks," in International Workshop on Ant Colony Optimization and Swarm Intelligence. Springer, 2006, pp. 49-59.

[16] Y.-f. Wen, Y.-q. Chen, and M. Pan, “Adaptive ant-based routing in wireless sensor networks using energy* delay metrics," Journal of Zhejiang University-SCIENCE A, vol. 9, no. 4, pp. 531-538, 2008 .

[17] R. Khoshkangini, S. Zaboli, and M. Conti, "Efficient routing protocol via ant colony optimization (aco) and breadth first search (bfs)," in Proceedings of the IEEE International Conference on Cyber, Physical and Social Computing (IEEE CPSCom 2014), 2014.

[18] Y. Sun, W. Dong, and Y. Chen, "An improved routing algorithm based on ant colony optimization in wireless sensor networks," IEEE Communications Letters, 2017.

[19] L. Xinlu, B. Keegan, and F. Japhet, "Ant colony clustering routing protocol for optimization of large scale wireless sensor networks,"IT\&T, 2015.

[20] A. Nayyar and A. Gupta, “A comprehensive review of cluster-based energy efficient routing protocols in wireless sensor networks," IJRCCT, vol. 3, no. 1, pp. 104-110, 2014. 
[21] A. Manjeshwar and D. P. Agrawal, "Teen: Arouting protocol for enhanced efficiency in wireless sensor networks." in ipdps, vol. 1, 2001, p. 189.

[22] O. Younis and S. Fahmy, "Heed: a hybrid, energy-efficient, distributed clustering approach for ad hoc sensor networks," IEEE Transactions on mobile computing, vol. 3, no. 4, pp. 366-379, 2004.

[23] S. P. Singh and S. Sharma, "A survey on cluster based routing protocols in wireless sensor networks,” Procedia computer science, vol. 45, pp. 687-695, 2015.

[24] F. Correia and T. Vazao, "Simple ant routing algorithm strategies for a (multipurpose) manet model," Ad Hoc Networks, vol. 8, no. 8, pp. 810-823, 2010. 